\title{
The ionospheric footprint of antiparallel merging regions on the dayside magnetopause
}

\author{
I. J. Coleman, M. Pinnock, A. S. Rodger \\ British Antarctic Survey, High Cross, Madingley Road, Cambridge, CB3 0ET, UK \\ e-mail: ijc@bas.ac.uk
}

Received: 15 November 1999 / Revised: 7 February 2000 / Accepted: 16 February 2000

\begin{abstract}
The antiparallel merging hypothesis states that reconnection takes place on the dayside magnetopause where the solar and geomagnetic fields are oppositely directed. With this criterion, we have mapped the predicted merging regions to the ionosphere using the Tsyganenko 96 magnetic field model, distinguishing between regions of sub-Alfvénic and super-Alfvénic magnetosheath flow, and identifying the day-night terminator. We present the resulting shape, width and latitude of the ionospheric dayside merging regions in both hemispheres, showing their dependence on the Earth's dipole tilt. The resulting seasonal variation of the longitudinal width is consistent with the conjugate electric fields in the northern and southern cusps, as measured by the SuperDARN HF radars, for example. We also find a seasonal shift in latitude similar to that observed in satellite cusp data.
\end{abstract}

Key words: Ionosphere (ionosphere-magnetosphere interactions) - Magnetospheric physics (magnetopause, cusp and boundary layers; magnetosphere-ionosphere interactions)

\section{Introduction}

The concept of magnetic reconnection at the dayside magnetopause, introduced by Dungey (1961), has become accepted as the most important process conditioning the interaction between the solar wind and the terrestrial magnetosphere. The potential drop across the magnetopause $X$-line is mapped to the ionosphere by newly reconnected field lines: the consequences of this projected merging line can be observed in enhanced flows, particle precipitation and polar cap boundary

Correspondence to: I. J. Coleman motion (Smith and Lockwood, 1996). In the present work, we use the terms reconnection and merging as synonyms, following Vasyliunas (1975).

The issue of where on the magnetopause reconnection occurs is still unresolved. The relative orientation of the magnetospheric and magnetosheath magnetic fields (the magnetic shear), the flow speed in the magnetosheath and the plasma $\beta$ in the magnetosheath are factors that may control reconnection. We employ the antiparallel merging hypothesis (Crooker, 1979) here, which states that reconnection takes place on those regions of the magnetopause where the magnetosheath and magnetopause fields are oppositely directed. This hypothesis has received observational support from twopoint spacecraft observations (Šafránková et al., 1998) and the phenomenology of the particle cusp (Newell et al., 1995). Our eventual aim is to test the antiparallel hypothesis by making a quantitative connection between magnetopause merging sites and observations of ionospheric signatures of reconnection. This work represents a first step in that direction. Note, however, that antiparallel merging with a purely southward IMF, the only case we discuss in the present study, is broadly equivalent to a sub-solar merging region (Gonzalez and Mozer, 1974), the other major proposal.

In order to make the connection between magnetopause merging and ground-based (or low-altitude) observations, it is necessary to employ a magnetospheric magnetic field model to map along the field lines from the magnetopause to the ionosphere. We have used the Tsyganenko 96 model (Tsyganenko, 1995; Tsyganenko and Stern, 1996) for two reasons. Firstly, it represents the current state-of-the-art in magnetospheric modelling. Secondly, it features an explicitly prescribed (ellipsoidal) magnetopause. This makes it well-suited to the modular approach demanded by this work, since it is at this boundary that the three main components of the model are joined (see Sect. 2).

Previous discussions of the merging site, ionosphere mapping have generally been of a qualitative nature (Crooker and Siscoe, 1990; Cowley et al., 1991; Lockwood, 
1997). More quantitative studies have been carried out by Maynard et al. (1995). Stasiewicz (1991) used the Tsyganenko 89 model to map the polar cusp to the ionosphere. The modelling that we present here is more quantitative than the former work, and broader in scope than the latter. In particular, Lockwood (1997) noted that Stasiewicz had neglected field line draping, and had virtually no open flux threading the dayside magnetopause. This study includes both these phenomena in the modelling. Our model of antiparallel reconnection sites follows that of Luhmann et al. (1984), albeit in a slightly simplified fashion.

In Sect. 2, we discuss the details of our modelling from the simple solar wind model to the identification of merging sites, then via the Tsyganenko 96 field model to the ionosphere. Section 3 shows the quantitative results. We first present ionospheric maps of the projected merging region for the March equinox. Then we show the effects of dipole tilt by displaying maps for the Northern and Southern Hemisphere ionospheric merging regions during the June and December solstices. The dipole tilt will be seen to have dramatic consequences upon the merging line widths and conjugate electric fields. Finally, we discuss the sensitivity of the results to the model parameters.

\section{Modelling}

Any model which maps magnetopause reconnection sites to the ionosphere should have three main components: field line draping in the magnetosheath, reconnection rate and magnetospheric magnetic field. For the sake of simplicity, we have used the "perfect draping" approximation: that is, the magnetosheath field is everywhere tangential to the magnetopause. This is a good approximation on the dayside, becoming less realistic further towards the tail. For our reconnection model, we adopt the antiparallel merging hypothesis: reconnection occurs in those regions where the magnetosheath and magnetosphere field are oppositely directed. Following Luhmann et al. (1984), we use the criterion that the fields must be within $10^{\circ}$ of being antiparallel. The reconnection rate is thus a simple step function, zero when the magnetic shear is less than $170^{\circ}$ and at its maximum when the shear is greater than $170^{\circ}$. Finally, we use the Tsyganenko 96 magnetospheric field model for the field-line tracing from the magnetosphere to the ionosphere. There is no time-dependence in the model.

The methodology is straightforward. First, we specify the solar wind conditions $\left(B_{y}, B_{z}\right.$, and dynamical pressure), $D_{s t}$ and the epoch (year, day and UT). These are the input parameters required by the Tsyganenko 96 model. Then we discretise a region on the magnetopause from the subsolar point to approximately $25 \mathrm{Re}$ tailward of Earth, beyond which the perfect draping approximation is unlikely to be applicable. The limit to this approximation will depend upon the solar wind parameters: analytic modelling of the magnetosheath field (Kobel and Flückiger, 1994) indicates that our chosen cutoff is reasonable for purely southward IMF with no $x$ component. At each point on this grid, we calculate the magnetic shear $\phi$. Where the merging criterion is fulfilled (here, $\cos (\phi) \leq-0.98)$, the field line is traced to the ionosphere. The end result is an array of points in the ionosphere (corrected geomagnetic latitudes and longitudes) with their associated merging angles and the GSM coordinates of the starting point on the magnetopause. This is used to construct the maps shown in the next section.

\subsection{Magnetopause regions}

There are two important boundaries on our model magnetopause, which divide the magnetopause into three distinct regions. The first is the Alfvénic boundary, where the sheath flow becomes super-Alfvénic. Rodger et al. (2000) argue, following La Belle-Hamer et al. (1995), that quasi-steady reconnection can only occur within the sub-Alfvénic region (between the subsolar point and the Alfvénic boundary), and that only transient reconnection is possible in the super-Alfvénic regime. In mapping the magnetopause merging line to the ionosphere, we are primarily interested in the quasi-steadystate reconnection that can occur in the sub-Alfvénic magnetopause region, and we argue that most of the reconnection potential generated will appear in this region.

The location of the Alfvénic boundary depends mainly upon the velocity of the solar wind. We have adapted the approach of Cowley and Owen (1989), who calculated that this boundary would lie at a distance of $6.8 R_{e}$ along the magnetopause from the subsolar point, based on a typical solar wind velocity of $500 \mathrm{kms}^{-1}$. Ignoring the curvature of the magnetopause between the subsolar point and the terminator, the $x$-coordinate $x_{a}$ of the Alfvénic boundary in the GSM system is

$x_{a}=r_{s}\left(1-r_{a} / \sqrt{\left(r_{s}^{2}+r_{d}^{2}\right)}\right)$

where $r_{s}$ is the subsolar stand-off distance of the magnetopause, $r_{d}$ is the radius of the dawn-dusk crosssection and $r_{a}$ is the distance along the magnetopause between the subsolar point and the Alfvénic boundary. Our input parameters give $r_{s}=10.3 R_{e}$ and $r_{d}=13.6 R_{e}$. Using the Cowley and Owen (1989) value for $r_{a}$ $\left(r_{a}=6.8 R_{e}\right)$ gives $x_{a}=6.2 R_{e}$.

The second boundary that we identify is the day/ night terminator. Given that the phenomenon of postterminator flux transfer events (time-varying reconnection) has been reported (Kawano and Russell, 1996), we identify where the magnetic field lines threading the day/ night terminator on the magnetopause map to the ionosphere. The teminator has no significance as a physical boundary: we show it purely as a guide to the reader, and to place post-terminator phenomena in context. Note, however, that it has yet to be shown that postterminator FTEs produce an ionospheric flow signature.

The whole section of the magnetopause that we consider is bounded by the subsolar point and by a prescribed cutoff far down the tail (at $x-25 R_{e}$ in GSM coordinates). 


\section{Results}

We have modelled epochs which cover the three extreme cases of the dipole tilt: 1700 UT on 21 June (dipole tilt $+34^{\circ}$ ), 2300 UT on 21 March (dipole tilt $0^{\circ}$ ) and 0500 UT on 21 December (dipole tilt $-34^{\circ}$ ). In all cases, we have chosen a southward IMF and typical values for $D_{s t}$ and the solar wind pressure. Specifically, $B_{y}=0, B_{z}=-5 \mathrm{nT}, D_{s t}=-20 \mathrm{nT}$ and $P_{d y n}=2.5 \mathrm{nPa}$.

\subsection{Magnetopause maps}

Figure 1 shows the extent of the antiparallel region on the magnetopause, for the three epochs considered in this study. The projection is in the $y-z$ GSM plane. The ' 10 ' contour shows the boundaries of this region (where the terrestrial and interplanetary fields are within $10^{\circ}$ of anti-parallel). The two ellipses show the Alfvénic boundary and the plane $X_{g s m}=0$ (the dayside-nightside terminator) respectively.

In all cases, the maps are symmetrical about $Y=0$. This is because, for all three epochs, the dipole axis is in the $x-y$ plane. At the equinox, when the dipole tilt is zero, the map is also symmetric about $Z=0$. This map corresponds to Luhmann et al. (1984) Fig. 2, for the IMF vector $(0,0,-1)$.
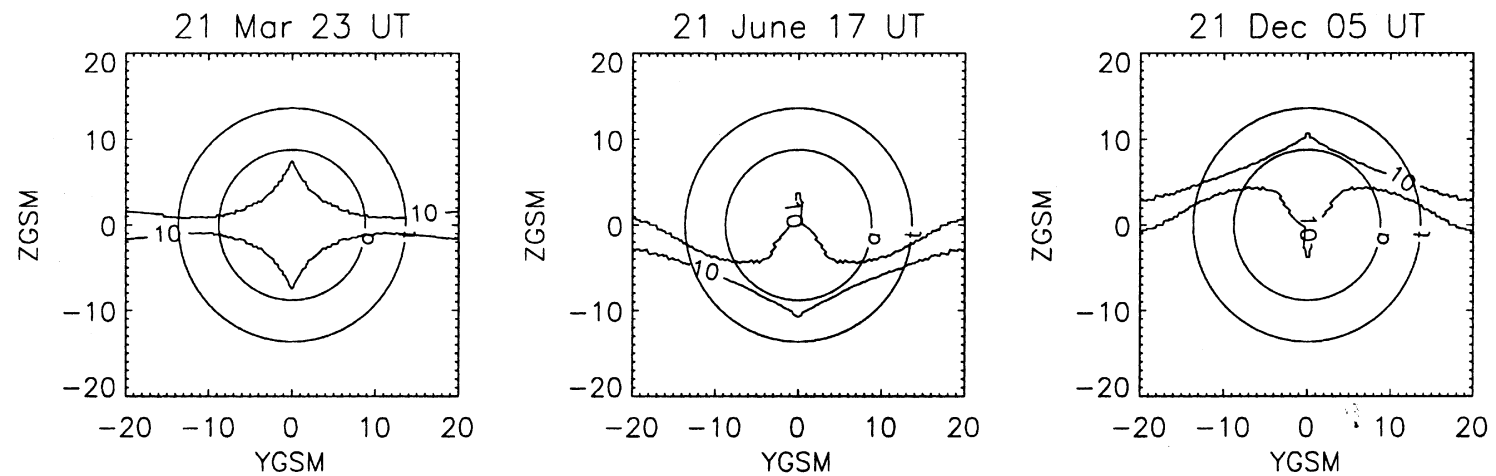

Fig. 1. Magnetopause maps in the $y-z$ GSM plane for the northern vernal equinox, northern winter and northern summer, under purely southward IMF. The ' 10 ' contour marks the region where the

terrestrial and interplanetary fields are within $10^{\circ}$ of being antiparallel. The elliptical contours mark the Alfvénic boundary (' $a$ ') and the dayside-nightside (0600-1800) terminator (' $t$ ')
In the northern winter, the antiparallel region moves above the equator, and at the December solstice a portion of the antiparallel region near magnetic noon is moved northwards past the Alfvénic boundary. During the northern summer, the situation is reversed, with the antiparallel region shifted south of the equator, and partially southwards of the Alfvénic boundary. The centroid of the reconnection region moves $\pm 5 R_{e}$ about the equatorial plane between solstices. The post-terminator regions are less affected by dipole tilt effects than the sub-solar region.

\subsection{Ionospheric maps}

Figures 2 to 4 show where the antiparallel region on the magnetopause maps to in the ionosphere, in magnetic latitude and longitude. The greyscale coding indicates whether the sub- or super-Alfvénic magnetopause region is mapping to that particular point in the ionosphere. The darker shading corresponds to the subsolar, sub-Alfvénic region, the lighter shading shows the super-Alfvénic region. A solid line indicates the dayside-nightside terminator; more precisely, the sections of the $X_{g s m}=0$ boundary on the magnetopause between $\pm 7 R_{e}$ either side of the equator, mapped to the ionosphere.

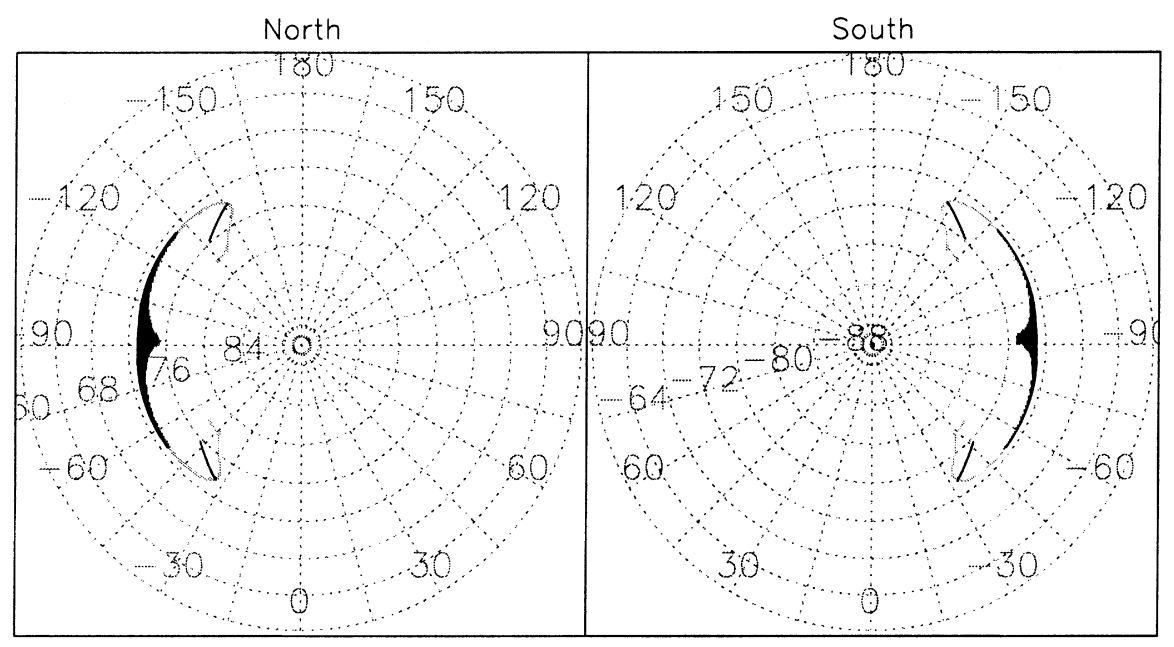



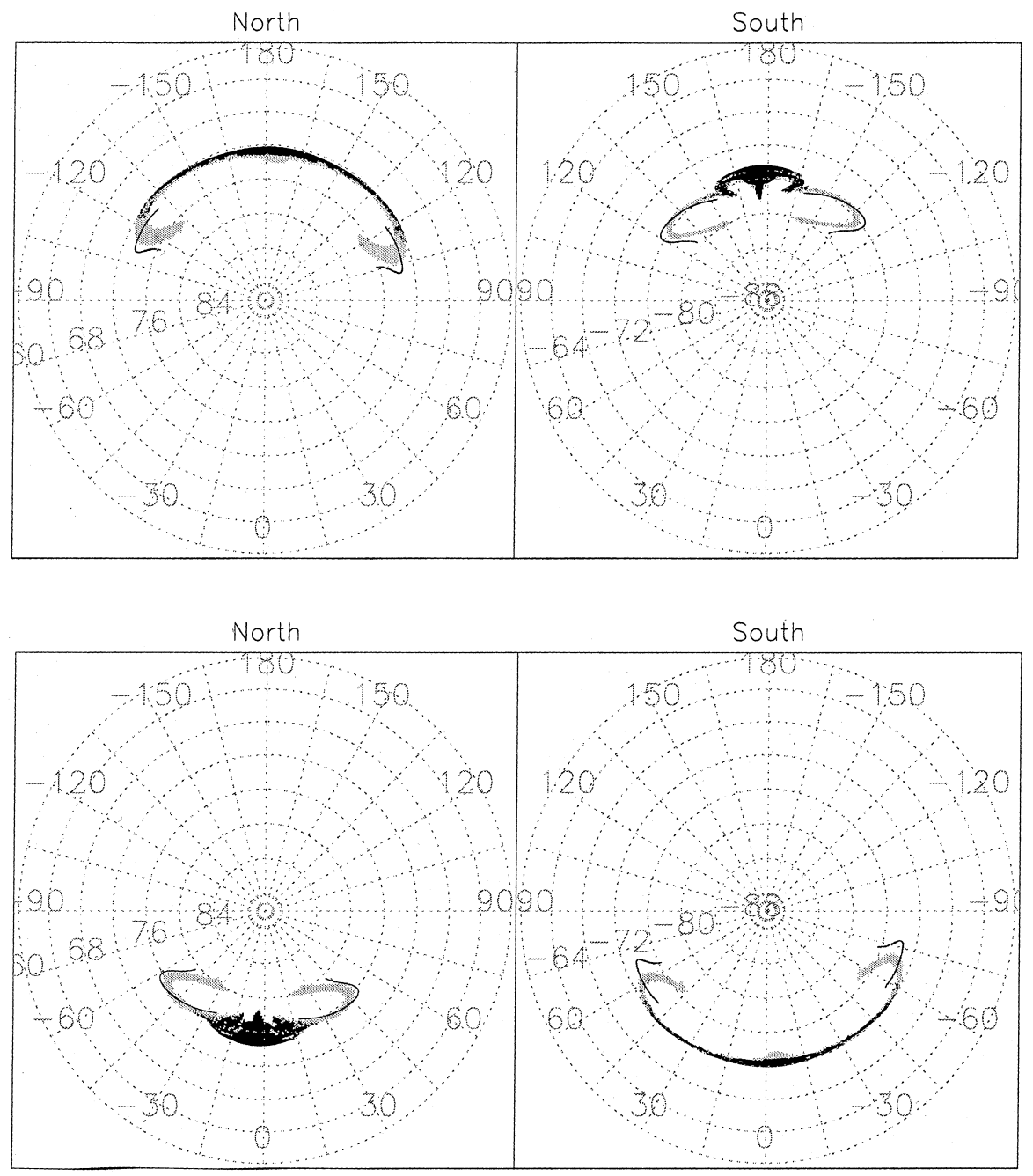

Fig. 3. Merging line at the northern winter solstice for $0500 \mathrm{UT}$. The coordinate system and greyscale shading scheme are as in Fig. 2
Fig. 4. Merging line at the northern summer solstice for $1700 \mathrm{UT}$. The coordinate system and greyscale shading scheme are as in Fig. 2
We show two map projections, centred on the North and South magnetic poles respectively. These projections are mirror images of each other, such that $+90^{\circ}$ longitude is on the right-hand side of the north polar projections, and on the left-hand side of the south polar projection.

\subsection{Merging line for southward IMF}

The first pair of maps (Fig. 2) display the results for the Northern and Southern Hemispheres at the northern vernal equinox. The two hemispheres show similar results, as might be expected in this case of zero dipole tilt. It can be seen that the latitudinal extent of the merging region is a maximum at magnetic noon and decreases in a symmetrical fashion either side of noon.

The poleward part of this wide central region maps to the high-latitude merging regions around $Y_{g s m}=0$ (as shown in Fig. 1). A field line passing through this highlatitude merging region will have the antiparallel condition satisfied along a large proportion of its total length. According to the antiparallel merging hypothesis, reconnection can occur anywhere along this section of the field line. If reconnection takes place at many places on the field line simultaneously, only the highestlatitude reconnection site will map to the ionosphere. This is consistent with the result of Newell et al. (1995), who noted that the altitude of the merging site is typically $8-12 R_{e}$.

The equatorward edge of this central region maps to the subsolar point. It should be emphasised here that the reconnection region has a finite thickness $(\sim 1$ ion gyroradius). At the subsolar point, the magnetospheric field lines are at their most compressed. Thus, field lines which are close together at the subsolar point, within the reconnection region, can map to ionospheric regions which are significantly separated in latitude. The field line closest to the Earth maps to the equatorward edge of the ionospheric footprint, while the field line furthest from the Earth maps to the poleward edge. However, the latter field line also passes through the high-latitude merging regions, as discussed, and only the highestlatitude reconnection site on this field line will produce an ionospheric signature.

The sub-Alfvénic region has a longitudinal extent of about $5 \mathrm{~h}$ (from $\sim-50^{\circ}$ to $\sim-135^{\circ}$ in the north). The post-terminator regions (along the flanks) map around $5^{\circ}$ poleward of the subsolar point, about 4 hours $\left(60^{\circ}\right)$ either side of noon. 
In Figs. 3 and 4 we show similar plots for the solstices, at times chosen to be the extrema of the dipole tilt angle. The extent of the sub-Alfvénic region is strongly dependent upon the dipole tilt. In the summer hemisphere, this region is compressed into a 'fat lip' about three hours across (in longitude) and a maximum of $4^{\circ}$ wide in latitude. In the winter hemisphere, by contrast, this region is thinner $\left(\sim 2^{\circ}\right)$, with a much greater longitudinal extent (around $8 \mathrm{~h}$ ). If most of the reconnection voltage is located within the sub-Alfvenic region, the conjugate ionospheric electric fields will be proportional to the extent of this region in each hemisphere. This proportionality will be most clearly seen at the solstices, when the dipole tilt is at its most extreme, but a smaller effect will be observable throughout the year. Indeed, this very effect has been observed by Pinnock et al. (1999).

This tilt angle dependency is mainly caused by the flaring out of the field lines on the magnetopause close to noon in the winter hemisphere, due to their experiencing lower solar wind pressure than in the summer hemisphere. The flank regions show much less dependency on the tilt angle, as was also seen in Fig. 1.

Note also that the equatorward edge of the merging region is about $2^{\circ}$ higher in latitude near noon in the summer hemisphere than in the winter hemisphere. As with the longitudinal extent, this is a result of the field lines in the summer hemisphere encountering greater solar wind pressure than those in winter. Such a seasonal shift in latitude has been observed by Newell and Meng (1989) and Zhou et al. (1999).

\section{Sensitivity to reconnection criterion}

Throughout this work, we have assumed that reconnection may take place provided the fields are within $10^{\circ}$ of being antiparallel. The choice of $10^{\circ}$ is arbitrary, but in fact the results are not sensitive to this number. Figure 5 shows the ionospheric merging region for a range of antiparallelness criteria, from $1^{\circ}$ to $20^{\circ}$, for the northern vernal equinox case. The shape and longitudinal extent of the $x$-line are unchanged, and the only effect of increasing the permitted deviation from perfect antiparallelness is to slightly increase the latitudinal thickness of the merging region, which is most marked in the subAlfvénic regime. This thickening is simply caused by the antiparallel condition being satisfied over a larger region of the magnetopause as the criterion is relaxed. The solstice cases (not shown in this study) are similarly insensitive to the precise reconnection criterion.

\section{Conclusions}

Using the antiparallel merging hypothesis, we have modelled the mapping of dayside magnetospheric reconnection sites to the ionosphere, in both hemispheres for a range of seasons, for southward IMF. We have shown that the dipole tilt angle has a dramatic effect on the ionospheric footprint of the dayside magnetopause. Specifically, the region where the reconnection rate is expected to be highest can be stretched by almost a factor of three in the winter hemisphere, as compared with the footprint in the summer hemisphere at the same instant. This leads to a prediction of greatly enhanced electric fields (by up to a factor of 3 ) in the summer hemisphere, which have been observed by the SuperDARN HF radar (Pinnock et al., 1999). This asymmetry of electric fields in the ionosphere needs to be incorporated into thermosphere-ionosphere coupled models, if the response to IMF forcing is to be properly captured.

The flanks map to regions several hours away from noon, with the post-terminator regions significantly poleward of the subsolar point. The size of these regions is less affected by dipole tilt than the subsolar regions. It
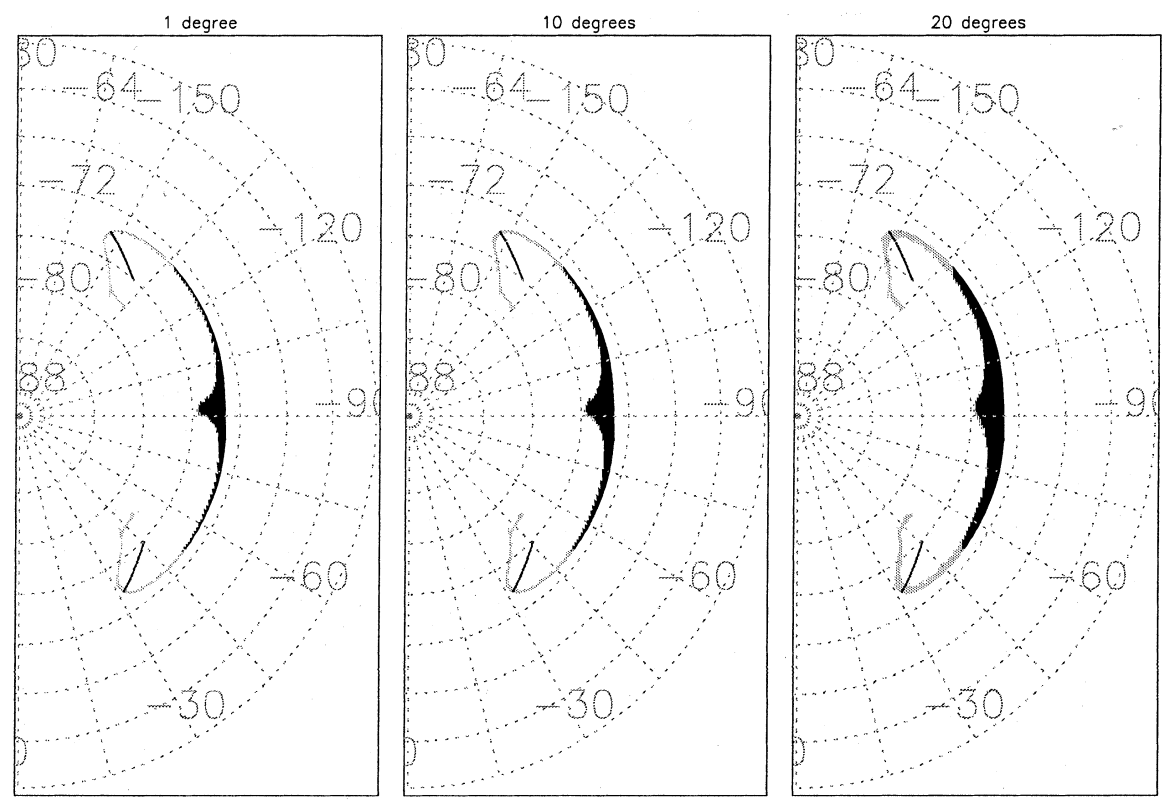

Fig. 5. The effect on the merging line of varying the antiparallel criterion, for the equinox case (see Fig. 2). As we increase the range of magnetic shears for which reconnection occurs in the model, the merging line increases in latitudinal extent. However, the effect is small, indicating that our results are not sensitive to the antiparallelness condition 
is here that the effects of post-terminator FTEs, if any, can be expected to appear in the ionosphere.

This work has focussed on the effects of dipole tilt angle during southward IMF. The next stage in the modelling is to consider the role of the solar wind: specifically, the IMF orientation and solar wind dynamical pressure.

Acknowledgements. We would like to thank N. Tsyganenko for making his model available to the community as a whole.

Topical Editor G. Chanteur thanks O. Santolik and P. Prikryl for their help in evaluating this paper.

\section{References}

Cowley, S. W. H., and C. J. Owen, A simple illustrative model of open flux tube motion over the dayside magnetopause, Planet. Space. Sci., 37, 1461, 1989.

Cowley, S. W. H., J. P. Morelli, and M. Lockwood, Dependence of convective flows and particle precipitation in the high-latitude dayside ionosphere on the $X$ and $Y$ components of the interplanetary magnetic field, J. Geophys. Res., 96, 5557, 1991.

Crooker, N. U., Dayside merging and cusp geometry, J. Geophys. Res., 84, 951, 1979.

Crooker, N. U., and G. L. Siscoe, On mapping flux transfer events to the ionosphere, J. Geophys. Res., 95, 3795, 1990.

Dungey, J. W., Interplanetary magnetic field and the auroral zone, Phys. Rev. Lett., 6, 47, 1961.

Gonzalez, W. D., and F. S. Mozer, A quantitative model for the potential resulting from reconnection with an arbitrary interplanetary magnetic field, J. Geophys. Res., 79, 4186, 1974.

Kawano, H., and C. T. Russell, Survey of flux transfer events observed with the ISEE 1 spacecraft: rotational polarity and the source region, J. Geophys. Res., 101, 27 299, 1996.

Kobel, E., and E. O. Flückiger, A model of the steady state magnetic field in the magnetosheath, J. Geophys. Res., 99, 23 617, 1994.

La Belle-Hamer, A. L., A. Otto, and L. C. Lee, Magnetic reconnection in the presence of sheared flow and density asymmetry: applications to the Earth's magnetopause, J. Geophys. Res., 100, 11 875, 1995.
Lockwood, M., Relationship of dayside auroral precipitations to the open-closed separatrix and the pattern of convective flow, J. Geophys. Res., 102, 17 475, 1997.

Luhmann, J. G., R. J. Walker, C. T. Russell, N. U. Crooker, J. R. Spreiter, and S. S. Stahara, Patterns of potential magnetic field merging sites on the dayside magnetopause, J. Geophys. Res., 89, 1739, 1984.

Maynard, N. C., W. F. Denig, and W. J. Burke, Mapping ionospheric convection patterns to the magnetosphere, J. Geophys. Res., 100, 1713, 1995.

Newell, P. T., and C.-I. Meng, Dipole tilt angle effects on the latitude of the cusp and cleft/low-latitude boundary layer, J. Geophys. Res., 94, 6949, 1989.

Newell, P. T., D. G. Sibeck, and C.-I. Meng, Penetration of the interplanetary magnetic field $B_{y}$ and magnetosheath plasma into the magnetosphere: Implications for the predominant magnetopause merging site, J. Geophys. Res., 100, 235, 1995.

Pinnock, M., A. S. Rodger, K. B. Baker, G. Lu, and M. Hairston, Conjugate observations of the day-side reconnection electric field: A GEM boundary layer campaign, Annales Geophysicae, 17, 443, 1999.

Rodger, A. S., I. J. Coleman and M. Pinnock, Some comments on transient and steady-state reconnection at the dayside magnetopause, Geophys. Res. Lett., (in press 2000).

Šafránková, J., Z. Němeček, D. G. Sibeck, L. Pech, J. Měrka, and O. Santolík, Two point observation of high-latitude reconnection, Geophys. Res. Lett., 25, 4301, 1998.

Smith, M. F., and M. Lockwood, Earth's magnetospheric cusps, Rev. Geophys., 34, 233, 1996.

Stasiewicz, K., Polar cusp topology and position as a function of interplanetary magnetic field and magnetic activity: comparison of a model with Viking and other observations, J. Geophys. Res., 96, 15 789, 1991.

Tsyganenko, N. A., Modelling the Earth's magnetospheric magnetic field confined within a realistic magnetopause, J. Geophys. Res., 100, 5599, 1995.

Tsyganenko, N. A., and D. P. Stern, Modelling the global magnetic field of the large-scale Birkeland current systems, J. Geophys. Res., 101, 27 187, 1996.

Vasyliunas, V. M., Theoretical models of magnetic field line merging I, Rev. Geophys., 13, 303, 1975.

Zhou, X-W., C. T. Russell, G. Le, S. A. Fuselier, and J. D. Scudder, The polar cusp connection and its dependence on dipole tilt, Geophys. Res. Lett., 26, 429, 1999. 